\title{
Classification of Neural Network with CT Images for Lung Cancer Detection
}

\author{
Uppuluri Rajasekhar, P.Jagadeesh
}

\begin{abstract}
ABSRACT-:This paper propose a clever method for the development to raise the over all performance of computeraid diagnosis and for computer, pulmonary nodule identity computed to mography snapshots that digital image and communication in the medicine formats. The preliminary segment in willpower of any variation from the norm in lung locale is to benefit a computer tomography photo.The advanced organization of the picture is very convenient, consequently the extraction and sharing of significant information. The substantial number of related pictures represent a test in soundness and thusly touching base at the end. The cad framework is planned and created to fragment the lung tumor district and concentrate on the highlights which are of the area of intrigue. The detection procedure involve of two stages, to be specific Lung division and feature extraction. In segmentation of lung region, fuzzy $c$ implied, GLCM primarily based algorithms are implemented. The extracted functions the design of comparable region of the internet use to teach the neural network. Finally these properties are used for analysis lung tumor as benign or malignant. The important goal for this method is reduce fake fantastic charge and to enhance the get entry to time and reduce interobserver variability.
\end{abstract}

KEYWORDS: DICOM, GLCM, Digital Images, Support Vector Machines, neural network, fuzzy c.

\section{INTRODUCTION}

A malignant growth is a phase of human body when the disease cell of human body get related in vast numbers and will, in general, develop wildly. Lung cancer is the one of its main sources for the death two people all through the world as given by the World Health Organization. Treatment of malignant growth is emotionally dependent on the physiology of the patient. The fundamental driver for the Lung Cancer is Tobacco Smoking. It has a frequency of this disease is far low in Female when contrast with Male. The information that is accessible all through the world will assist us with uncovering important data in regards to the example of rate. Lung disease starts discreetly. There are generally no other side effect or cautioning signs in the beginning periods. A winding CT may get early lung malignancies in some people. The US Preventive Services Task Forces prescribe yearly screening for Lung diseases with low-portion ct in grown-ups somewhere between the range of 55 and 80 years who have 30 pack year smoke history, and at present smoking have stopped inside previous 15 years,the most seasoned portrayal of

Revised Manuscript Received on August 14, 2019.

Uppuluri Rajasekhar, UG Scholar, Department of ECE, Saveetha school of engineering, Saveetha Institute of Medical and Technical Science, Chennai, TamilNadu, India(email: rsekhar617@gmail.com)

P.Jagadeesh, Assistant Professor, Department of ECE, Saveetha school of engineering, Saveetha Institute of Medical and Technical Science, Chennai, TamilNadu, India(email: jagadeeshp@ saveetha.com) malignancy was found in Egypt. Bone Cancer named as osteosarcoma have been seen in mummies. Studies have described that the utilization of CT improves trust, in conclusion, expands the quantity of the "authoritative" injuries by $41 \%$ in patients with non-little cell lung disease.

In PC vision the image division is of the path toward parceling of a computerized picture into the various fragments. The objective of the division to improve and additionally change the portrayal of the image into something increasingly important and simpler to investigate.

\section{SYSTEM ARCHITETUTE}

The time period automatic images mention for preparing the two dimensional picture by a sophisticated PC. In an extra tremendous putting, it infers computerized coping with any dimension facts.The advanced images for diffusion of actual or complex number spoken to by way of a confined range of bits. A image gives a straight forward, slide photograph and X-beam first digitizes is placed as a grid for twofold digit in computer reminiscence. This digital photograph then able to handle along with confirmed on an excessive-desires TV display screen. For this showcase, the image is taken a quick accessible cushion memories, invigorate screens at the rate of 25 outline for the second to delivers the constant presentation.

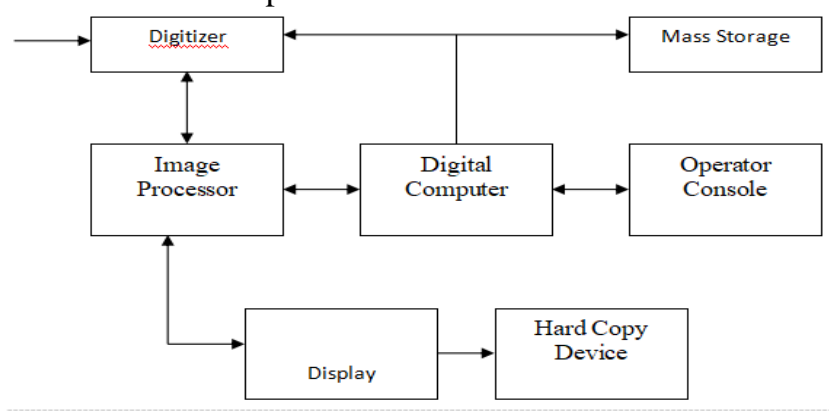

Digitizer:

A digitizer change over a image into the numerical Portrayals applicable for improvement to a computerized PC. Some regular digitizer

1. microdensitometer.

2. flying spot scanner.

3. image dissector.

4. videocon camera.

5. photosensitive solid-state arrays. 


\section{Image Processor}

Image processor is capabilities of the Image acquisition, garage, preprocessing, segmentation, representation, recognition, and the interpretation last presentations or data ensuing picture. This block diagram allows fundamental arrangement involves in the image processing system.

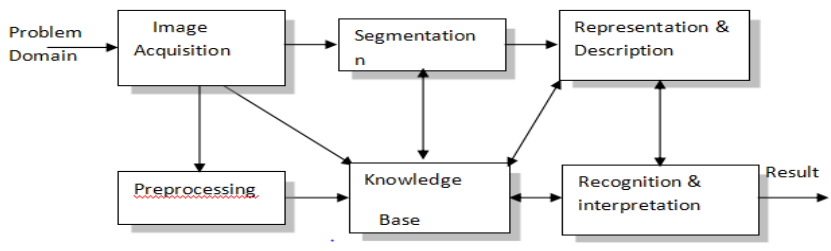

Detection and Classifications Lung Image Modality.Goal of this work is to settle on a choice emotionally supportive network for the recognizable proof and arrangement of the inspiratory knob in the lung picture as either dangerous or kindhearted tumor.

So as to conquer these issues, various strategies have advanced in the choice emotionally supportive network to lessen the bogus positive rate and subsequently to group the knob as destructive or non-dangerous. In this proposed framework the goal is to give quick recognizable proof and exact robotized division and thereof decrease the bogus positive rate utilizing characterization methods with lung Image datasets. The organization of lung CT picture database is DICOM design which is exposed to additional handling to limit for mistake rate.

\section{Image Processing Fundamental}

The digital image Processing refer to process to the images for the digital forms. Latest camera additionally immediately take the pictures virtual form however usually pics originated optical forms.Then they taken through camera and digital it.The digital process involves Sampling and Quantization. Later those pictures processed by using the 5 fundamental tactics, at least someone of them are not required for all of them.

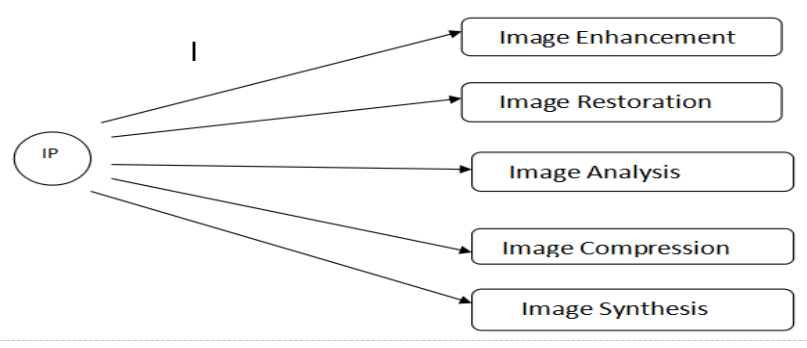

\section{1)Image Enhancement}

Image enhancement improves characteristics an image and enhance the images evaluation traits, reduce the noise material,and sharpen the info.This improves the images and well-known shows equal information the extra comprehensible photo. It no longer upload any data.

\section{2)Image Restoration}

Image restoration enhances quality of the photograph but operation is especially primarily based on acknowledged measured or decline the unique picture.Image restoration used to repairs photographs with issues together with the geometric distortion, mistaken cognizance, repetitive noises, digicam motion. It usesf or correct photographs to known degradation.

\section{3)Image Analysis}

Images evaluation operation produces numericals and graphical statistics base on traits the authentic photograph They destroy into the object and classified it. Then it rely the images statistic. operation are for extractions and the descriptions for the scenes and photo capabilities, automatic measurements and the objects type. Images analysis for the specifically utilized in the machine vision packages.

\section{4)Image Compression}

The Image compression and the decompression reduces information contained vital for explaining the image. Most of the images contained in it has a lot of redundant record, Because of this compressions, dimensions are reduced, successfully saved in it and transported in this. compressed photograph decompressed whilst display. Lossless compression preserve exact facts in original photo, however Loss compression does not represent unique picture however provide high-quality compression.

\section{5)Image Synthesis}

Image Synthesis operation creates pix different photographs and non-photographs statistic. Image synthesis operation typical photos which can be either physically not possible or impractical to collect.

\section{Objective:}

This paper, we have propose a unique method that detect and categorize fuzzy c-imply, GLCM(Gray degree coincidence matrix, The KNN (Knearest neighbor), svm (Support vector device) and neurals community is responsible for the lung cancer from the lung digital snapshots. This reduces the variability in the detection of the way of computerized segmentation and class of tumor. Evaluate with Neural network.

\section{Existing System}

This gives a way to concurrently determine the range of visible layers in the outer retina and section them. The approach is based on a version selection technique with special attention given to stability the best of a healthy with model complexity. As such, the model choice manner guarantees that a greater complicated model is selected handiest if sufficiently supported with the aid of the information. The approach is capable of deal with layers that may or won't be a gift inside a photograph and offer not best the wide variety of visible image, but also their role and identity.

\section{Proposed method :}

In this paper, we file the set-up and consequence of the Lung cancer prepared in Machine getting to know. Twenty present day segmentation algorithms were carried out to hard and fast of 65 multi-comparison MR scan of the low and excessive grades. tumor sufferers manually annotated by the way of as much as 4 raters and to sixty-five

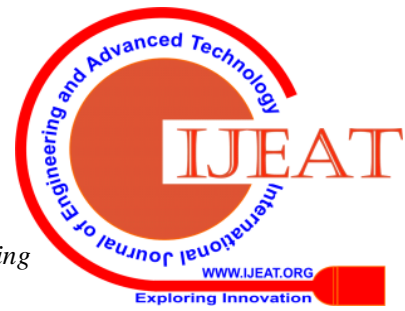


comparable scans generated the use of tumor photo simulation of the software. Quantitative opinions revealed tremendous war of words among the human rater in segmenting he diverse of the tumor sub-areas. Illustrating the issue of this mission. We located special algorithms labored high-quality for exclusive sub-areas however, that no of single set of rules ranks the inside the top for all the sub-areas concurrently. Fusing several true algorithms the usage of a hierarchical majority of the votes yielded the segmentation so that constantly ranks fantastically individual algorithm, indicating the remaining possibilities for the similarly in methodological improvements. The CT photo records and guide annotations stay publicly available through the web evaluation of the system as of the on going lung most cancers aid. Most of Data mining techniques used specifically to types of type techniques that are:

1. Statistical techniques

2. Data comparisons

- $\quad$ Statistical strategies along with, SVM and KNN; these methods specifically are very complex in making use of and in computation complexity.

- Data comparisons technique including Decision Tree, Nearest Neighbors, and Neural Network; those strategies are considered to be simple but on the equal time it is consumed a whole lot of time which boom the time complexity in those algorithms.

\section{SYSTEM DESIGN}

$\begin{array}{ll}\text { Algorithm/Method Implementation } \\ \text { 1. } \\ \text { 2. } & \text { GOUGH FUZZY C-MEAN } \\ \text { 3. } & \text { SVM } \\ \text { 4. } & \text { K-NN } \\ \text { 5. } & \text { NEURAL NETWORK }\end{array}$

\section{Method Implementation}

\section{Rough Fuzzy C-Mean}

Adaptation of tough fuzzy c-method set of rules for excessive dimensional facts by means of editing its objective feature. The proposed set of rules robotically detects the relevant cluster dimensions of the high dimensional facts set. The challenge of weights to attributes being specific to each cluster, a green subspace clustering scheme is generated.

\section{Feature Extraction:}

The proposed work uses GLCM and shape features for feature extraction.

\section{Glcm Feature Extraction:}

The co-incidence matrix and texture functions had been first of all used for the automatic classification of rocks. From then at the GLCM has been one of the typically used tools for texture evaluation due to the fact it can estimate photo homes associated with $2 \mathrm{~d}$-order facts. A picture with the size of pixels and gray degrees may want to illustrate the frequency of pixel incidence with gray level and according to with a distance $d$ from a certain pixel at the position with a grey degree. proposed fourteen measures of the textural capabilities that are derived from the co-occurrence matrix a famous statistical methods for the texture and feature extraction. It incorporates information about how the images intensities in the pixel with the positive positions in terms of every different occuring together. The texture one of the vital defining the characteristics of a photo. The gray degree co-incidence matrix is the 2-dimensional matrix of joint possibilities ) among pairs of pixels separated with the aid of a distance in a given path. The 2nd order photograph histogram called the Grey Level Co-occurrence Matrix (GLCM) of a photo offers extra information about the interpixel relationship, periodicity, and spatial grey degree dependencies. This matrix is a source of fourteen texture descriptors.

\section{Multi- SVM Classification:}

Supervised gaining knowledge of method is performed uses SVM classifiers for similarly lessen FPS. On the characteristic bases SVM classifier relies the series of the functions extracted each from the last INC after the rules based filter operations.

\section{KNN Classification:}

The KNN binary (as two class) is given more accurate data classification which beneficial to select $\mathrm{k}$ as an odd number which avoids the irregular data. The KNN procedure is the technique in ML procedures: It is an object which classified through a mainstream selection for its neighbors, with determination assigned occurrence for most mutual class among $\mathrm{k}$ nearest of the neighbour $(\mathrm{k}$ is the positive integer, classically tiny). Classically Geometer distances to use for distance metrics; however, it is only suitable for endless variable present in this. In such a situation as the classification of text, alternative metric, intersection metric and Hamming distances is to use. KNN is a new process that deliveries all available cases and categorizes novel cases built on an evaluation quantity (e.g., distance functions). KNN procedure is identical simple. It works built on the minimum distances from the interrogation instances for training the samples to regulate to $\mathrm{K}$ nearest neighbor. The information for $\mathrm{KNN}$ procedure contains numerous attribute which will be used for the categorize. The information of the KNN be any of the dimension scales from the insignificant, to a measurable scales.

\section{NEURAL NETWORK}

This proposes work uses the capabilities extracted from the preceding step as entering to ANN to categorize whether or not it's miles benign or malignant. Neural networks or connectionist structures are a computational version utilized in pc technological know-how and other studies disciplines, that's based on a big series of easy neural devices (synthetic neurons), loosely analogous to the discovered behavior of a biological mind's axons. Each neural unit is attached with many others, and hyperlinks may be decorated or inhibit the activation state of adjacent neural devices. Each man or woman neural unit computes usage of the summation characteristic. The phrase community inside the terms of the 
synthetic neural networks refers back to interconnections between neuron in special layer of each machine. An example machine has 3 layers. The first layer have input neuron which will sends the facts through synapse to second layer of the neuron, and through greater synapse to $1 / 3$ layer of the output neuron. complexs structures can has extra layer of neuron, a few have the accelerated layer of the input neuron and the output neuron. synapses save parameter known the weights control facts inside the calculation. An artificial neural network is a facts process example this stimulated by manner natural neural structures, consisting of the mind, method statistics. The fundamental element in this example of new shape records process machine. It combine big quantity fantastically process the element working accord to resolve the precise issues. ANN, similar humans, research examples. ANN is designed for specific package, along with pattern quality or facts categorization, finished the learning method. Acquisition in the organic system includes changes to conjunction relation between the neurons. this can be very of the ANN as properly

\section{MODULE NAMES}

\section{$>\quad$ PREPROCESSING \\ $>$ SEGMENTATION \\ $>$ FEATURE EXTRACTION}

\section{Preprocessing}

The reason of the preprocessing degree is to take away undesirable consequences together with the noise from photo and the transform and regulate photograph as the vital further process. The resolution of the photograph is decreased via the issue of 4 to $512 \cdot 384$ to hurry up the overall performance of the gadget. Also, the take a look at pics might be the subject, to the selective median filtering, and the unsharp covering to the isolated noise can also be amassed throughout picture acquisition and due to immoderate staining.

\section{Segmentation}

Methods for partition the photograph as a section could be described the photograph segmentation. Considee the similarity properly, the segmentation is carried out. This similar asset the clustering together which approachs implement Lloyd clustering techniques that aid inside segmentation of the blood microscopic picture on the basis of which alike homes. method broaden okay-imply clustering algorithms by which introdues the repeat segmentation of the schemes which are explore centroid for each set in segmention and sooner or later resection of the input totally at close centroid. This approach aid inside extraction of the important images traits, primarily based upon which the information can without difficulty perceived. simple thresholding approach implemented to the present initial label to the pixels within bloodmobile snapshots. set of rules is bases totally on apriori statistics blood smear pictures. Then the label which are adjust with the shape for detection technique based upon big region Alcon text information to provide significant outcomes.

\section{Feature Extraction}

sample popularity in photo process is the feature extraction of an unique form of dimensionality reduces The entered information is a set of rules is just too huge to process and the miles suspectes to notoriously redundanted, the enter records might transformed into the reduced illustration set of the feature. Transform the entered information into a set of the capabilities are known as function extraction. If the feature extraction is carefully chosen its miles expect capabilities will extract the applicable facts from entering the data with a purpose to perform preferred tasks the uses the reduce illustration as opposed to the overall length inputs. Features extraction includes simplify the number of resource required for explain the massive set of the records as it should be. When acting analysis of complicated information of predominant problem stem from variety of the variables concerned. Analysis with the huge number of the variables generally calls for the big quantity of the memory and the computation energy or the category algorithms which are over suits the training of the pattern and generalizethe poorly to the new sample. Feature extraction is the trendy time period which strategies the construction combination for the variables to around those problems and nevertheless describe the facts withthe sufficient accuracy.

\section{Module And Function}

\section{Preprocessing}

Reason for the preprocessing degree is do away with not wanted outcomes together with the noise from photo, and remodel and alter picture play vital similarly process. The decision of picture is reduced aspect of 4 to $512 \cdot 512$ speed up the overall performance of gadgets. The test pix might be the subject to the selected median filter and the unsharp overlaying to the isolated noise which will additionally were gathered during the image acquisition and due to the excessive staining

\section{Segmentation}

- The accurate segmentation of the lung area are essential given that, nodule gift the boundaries of the lung parenchyma. Such lung nodule lost and it reduce the sensing accuracy, if total lung isn't always segmention appropriately.

- Closing aim of the lung location interest segmentation for setting apart voxel similar to the lung place to the voxel similar to the encompassing material body.

- In this paper, fuzzy c-way algorithm were used as an effective for the segmentation of the CT lung picture.

\section{Feature Extraction}

- A GLCM capabilities are extracted from segmented lung area to uniquely specify areas.

- A GLCM is a largely a matrix in which range of the gray stages in the photograph equal to the wide variety of the rows and columns. 


\section{Classification}

- The GLCM capabilities are extracted are fed into neural network for type.

\section{Technique}

- In our approach, fuzzy c-way clustering is used to section the location of interest gift within the CT lung snap shots.

- The photograph information in 2-D is transformed into pixel data then fuzzy c-method algorithm. The fuzzy set of rules work as follows

- $\quad$ Choose some of clusters.

- Assign coefficients randomly to every information point for being within the clusters.

- Repeat till the set of rules has converged (that is, the coefficients' trade between two iterations is no greater than the given sensitivity threshold) :

- Compute the centroid for every cluster .

- For each facts factor, compute its coefficients of being in the clusters

- After segmentation a few morphological operation like remaining, dilation are carried out to put off unwanted small regions.

- Glcm functions extracted from lung nodule are fed into neural network for type

\section{RESULTS}
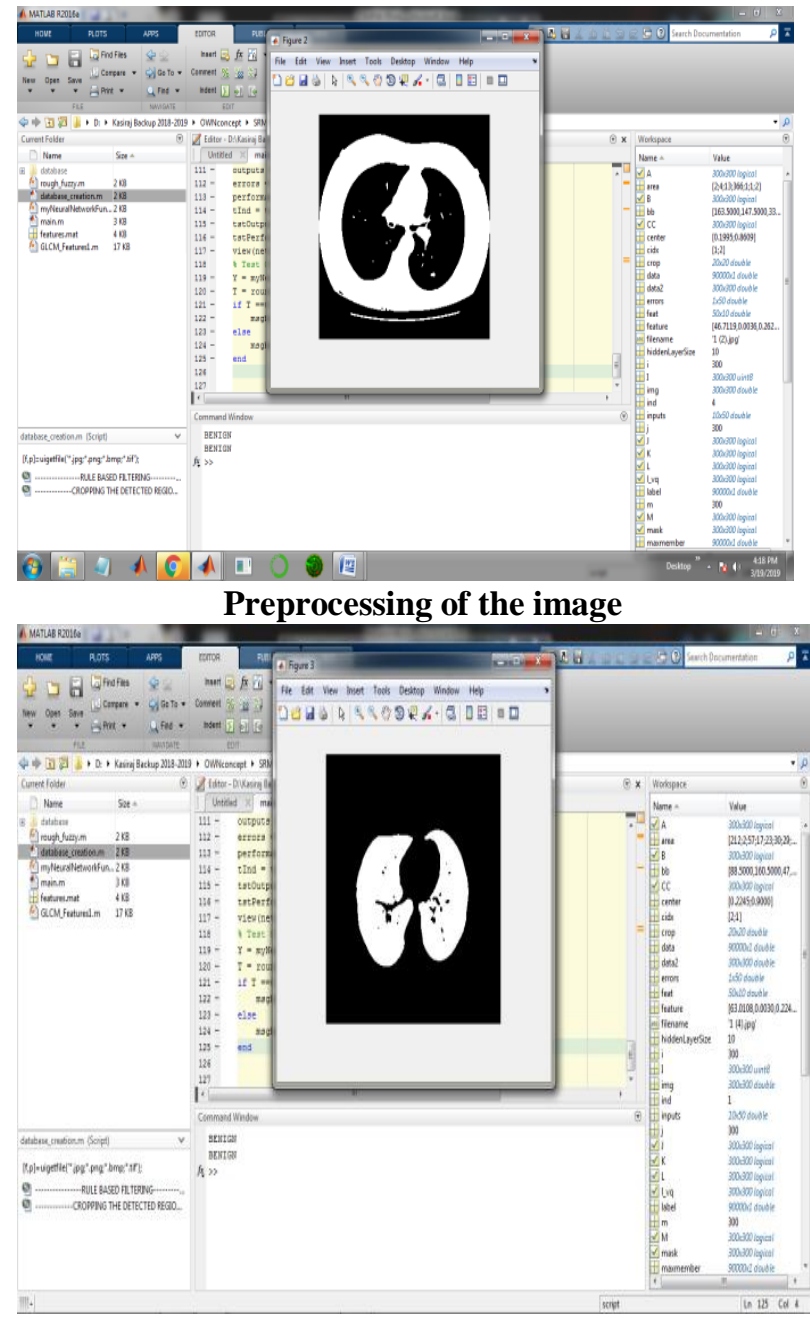

Segmentation of the image

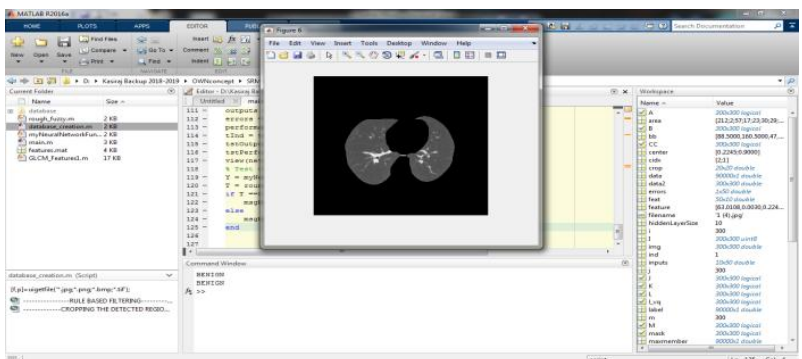

Feature exraction of the image
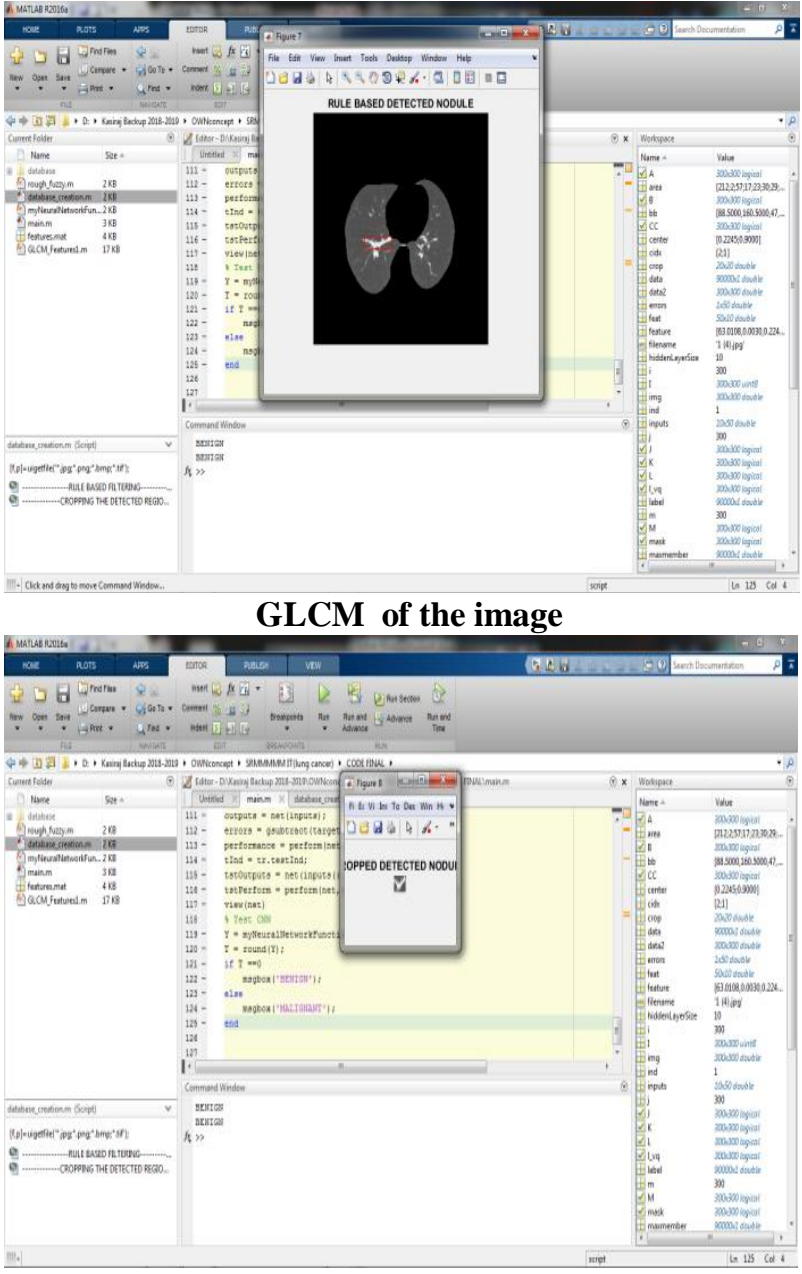

Detection of the nodule

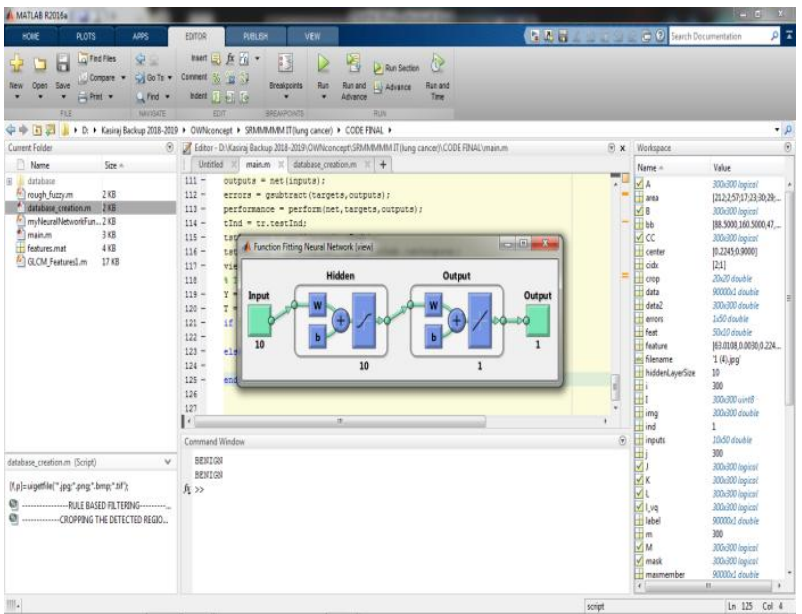

Fuzzy C clustering of the image

Published By:

Blue Eyes Intelligence Engineering

\& Sciences Publication

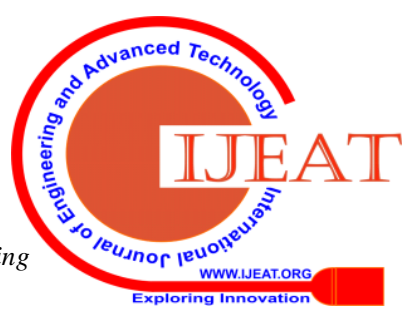




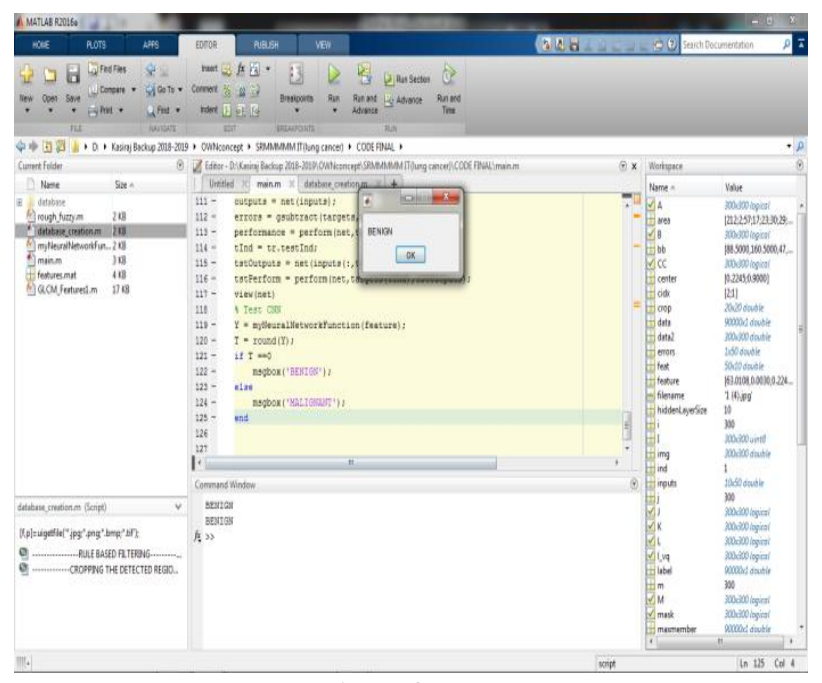

Detection of the tumour

\section{CONCLUSION}

This paper proposes the way to the automation segmentation, characteristic extraction and class of purple and white blood cells using KNN, NN, and SVM classification set rules. Several enhancements have been made to the SVM set of rules, along with the initialization steps for locate 12-neighbour linked aspect. With addition to it, the propose version feature the superior accuracy for selecting perfect circles from the three candidates circles, the functionality to discover abnormal cells, the use of a dynamic number of the iterations, advanced identification of the overlapping cell. It proposes approach accomplished segmentation. categories of which the BENIGN or MALIGNANT properly while effects have been compared, which turned into determined with the aid of specialists.

\section{REFERENCES}

1. J. Quintanilla dominguez, B. Ojedamagaña, M. G. Cortinajanuchs, R. ruelas, A. Vegacorona, and D. Andina, "Image segmentation by fuzzy and possibilistic clustering algorithms for the identification of microcalcifications," sharif university of technology scientia iranica , vol. 18 , pp. 580-589, Received 21 july 2010; revised 26 october 2010 accepted 8 february 2011.

2. Murat -ceylan, Yuksel ozbay, O. Nuri, ErkanYildirim, 2010, A novel method for the lung segmentation on chest CT images: complexvalued artificial neural network with complex wavelet transform, Turk JElec Eng \& Comp Sci, Vol.18, No.4 pp. 613-623.

3. FaizalKhan, Z\&Kavitha, V 2012, Estimation of objects in computed tomography lung images using supervised contextual clustering, research journal of the applied sciences, vol 7, no 9- 12, pp.494-499.

4. AntonelliM, LazzeriniB, MarcelloniF Segmentation and Reconstruction of the lung volume in CT images. 20th annual ACM symposium on applied computing, vol I. Santa Fe, New Mexico, pp. 255 -259, March 2005.

5. Salli, Hannu, J,Aronen, SauliSavolainen, AnttiKorvenoja \& Ari Visa 2001, contextual clustering for analysis of functional MRI data, IEEE transactions on Medical Imaging, vol. 20, no 5,pp.403-414, 2001.

6. FaizalKhan, Z \&Kannan, Intelligent Segmentation of Medical images using Fuzzy Bitplane Thresholding, Measurement science and Review, Vol 14, No 2 pp-94101, 2014.

7. JiantaoPu, JustusRoos, ChinA.Yi, SandyNapel, GeoffreyD.Rubin,David S. Paik, Adaptive Border
Matching Algorithm: Automatic Lung Segmentation on Chest CT images,Comp Medical Imaging and Graphics vol 32pp. 452-462, 2008.

8. GiorgioDeNunzio, EleonoraTommasi, AntonellaAgrusti, RosellaCataldo, Ivan DeMitri, MarcoFavetta, SilvioMaglio, AndreaMassafra, SabinaTangaro, PieroCalvini,NiccolòCamarlinghi,FabioFalaschi,Piergior gioCerello,and Piernicola Oliva, Automatic lung segmentation in CT images with accurate handling of the hilar region, Journal of digital imaging, Vol 24, Nolpp 11-27, 2011.

9. LinDT, YanCR, ChenWT, Autonomous Detection of Pulmonary Nodules on CT Images with a Neural Network-Based Fuzzy system, Comp Medical. Imaging and Graphics , Vol 29pp. 447-458, 2005.

10. A. A. Brindha, S. Indirani, and A. Srinivasan, "Lung cancer detection using SVM algorithm and optimization techniques," Journal of Chemical and Pharmaceutical Sciences, vol. 9, no. 4, 2016.

11. M. Kurkure and A. Thakare, "Introducing automated system for lung cancer detection using Evolutionary Approach,"

12. P. Bhuvaneswari and A. Brintha Therese, "Detection of cancer in lung with K-NN classification using genetic algorithm," International Conference on Nanomaterials and Technologies, vol. 10, pp. 433-440, 2014.

13. K. Venkatalakshmi and S. Mercyshalinie, "Classification of multispectral images using support vector machines based on PSO and k-means clustering," in Proceedings of IEEE International Conference on Intelligent Sensing and Information Processing, pp. 127-133, Bangalore, India, December 2005.

14. N. Panpaliya, N. Tadas, S. Bobade, R. Aglawe, and A. Gudadhe, "A survey on early detection and prediction of lung cancer," International Journal of Computer Science and Mobile Computing, vol. 4, no. 1, pp. 175-184, 2015.

15. Ilya Levner, Hong Zhangm ,Classification driven Watershed segmenta-tion, IEEE TRANSACTIONS ON IMAGE PROCESSING VOL. 16,NO. 5, MAY 2007 